\title{
Lie symmetries of nonrelativistic and relativistic motions
}

\author{
Carles Batlle* \\ Departament de Matemàtiques and IOC, Universitat Politècnica de Catalunya EPSEVG, \\ Avenida V. Balaguer 1, E-08808 Vilanova i la Geltrú, Spain \\ Joaquim Gomis ${ }^{\dagger}$ \\ Departament de Física Quàntica i Astrofísica and Institut de Ciències del Cosmos, \\ Universitat de Barcelona, Martí i Franquès 1, E-08028 Barcelona, Spain
}

\author{
Sourya Ray \\ Instituto de Ciencias Físicas y Matemáticas, Universidad Austral de Chile, 5090000 Valdivia, Chile \\ Jorge Zanelli ${ }^{\S}$ \\ Centro de Estudios Científicos (CECs), Avenida Arturo Prat 514, 5110466 Valdivia, Chile
}

(Received 23 January 2019; published 15 March 2019)

\begin{abstract}
We study the Lie symmetries of nonrelativistic and relativistic higher order constant motions in $d$ spatial dimensions, i.e., constant acceleration, constant rate-of-change-of-acceleration (constant jerk), and so on. In the nonrelativistic case, these symmetries contain the $z=\frac{2}{N}$ Galilean conformal transformations, where $N$ is the order of the differential equation that defines the constant motion. The dimension of this group grows with $N$. In the relativistic case the vanishing of the $(d+1)$-dimensional spacetime relativistic acceleration, jerk, snap, ..., is equivalent, in each case, to the vanishing of a $d$-dimensional spatial vector. These vectors are the $d$-dimensional nonrelativistic ones plus additional terms that guarantee the relativistic transformation properties of the corresponding $(d+1)$-dimensional vectors. In the case of acceleration there are no corrections, which implies that the Lie symmetries of zero acceleration motions are the same in the nonrelativistic and relativistic cases. The number of Lie symmetries that are obtained in the relativistic case does not increase from the four-derivative order (zero relativistic snap) onwards. We also deduce a recurrence relation for the spatial vectors that in the relativistic case characterize the constant motions.
\end{abstract}

DOI: 10.1103/PhysRevD.99.064015

\section{INTRODUCTION}

The study of the symmetries of nonrelativistic and relativistic motions in flat spacetime has been the subject of interest through the years; see [1,2]. In particular, the motions with constant acceleration in the relativistic case are at the basis of the Unruh effect [3]. The generalization of jerk and snap has attracted recent interest [4-6].

The Lie symmetries associated with a system of differential equations

\footnotetext{
*carles.batlle@upc.edu

joaquim.gomis@ub.edu

ray@uach.cl

§ @ cecs.cl
}

Published by the American Physical Society under the terms of the Creative Commons Attribution 4.0 International license. Further distribution of this work must maintain attribution to the author(s) and the published article's title, journal citation, and DOI. Funded by SCOAP.

$$
L_{a}\left(t, q^{A}, \frac{d q^{A}}{d t}, \ldots, \frac{\mathrm{d}^{n} q^{A}}{\mathrm{~d} t^{n}}\right)=0
$$

for $a=1, \ldots, r, A=1, \ldots M$, can be understood as those total, or passive, transformations

$$
q^{A} \rightarrow \tilde{q}^{A}=q^{A}+\delta q^{A}(t, q), \quad t \rightarrow \tilde{t}=t+\delta t(t, q)
$$

that map solutions of (1) into other solutions of (1) [7]. In order to determine these transformations it is useful to consider the associated functional, or active, variation of $\bar{\delta} q^{A}$, defined as

$$
\bar{\delta} q^{A}=\delta q^{A}-\dot{q}^{A} \delta t
$$

where the dot denotes the derivative with respect to $t$. Using the fact that the functional variation $\bar{\delta}$ commutes with the derivative with respect to $t$, the above symmetry requirement is equivalent to the demand that $\bar{\delta} q^{A}$ satisfies 


$$
\left.L_{a}[t, \bar{\delta} q]\right|_{L[t, q]=0}=0, \quad a=1, \ldots, r .
$$

This results in a set of partial differential equations for $\delta q(t, q)$ and $\delta t(t, q)$, which, in the more complex cases, can be solved with the help of algebraic computer packages [8].

We only consider spacetime Lie symmetries; that is, we do not admit the presence of derivatives of the space coordinates in the right-hand sides of Eq. (2). As we will discuss later, this allows us to interpret the symmetries in terms of transformations between equivalent observers. From the mathematical point of view one could, however, remove this restriction and consider a wider class of transformations, that is, Bäcklund-Lie symmetries of the equations of motion depending up to derivatives of the space coordinates of one order less than the order of the equations.

In the nonrelativistic case it has been proposed $[9,10]$, that the symmetry algebra of those motions contains the family of Galilean conformal groups [11,12]. One of the motivations of this work is to understand if there exists a family of relativistic conformal algebras that are a symmetry of the motion of zero jerk, zero snap, and their generalizations [5]. In this context we will often meet the $N$-Galilean conformal algebra (NGCA) [9-13]. In $d+1$ spacetime dimensions the NGCA has dimension

$$
3+d(N+1)+\frac{d(d-1)}{2},
$$

where the 3 corresponds to time translations, dilatations, and expansions that form the $\operatorname{Sl}(2, R)$ group, the last term counts the rotations in $d$ space, and the intermediate term $d(N+1)$ corresponds to space translations, ordinary Galilean boosts, and $N-1$ generations of higher order Galilean boosts, all in $d$ space.

Through this paper we will consider continuous transformations of a differential equation as spacetime symmetries in $d+1$ dimensions connecting two observers which describe a particle with constant position, constant velocity, constant acceleration, and so on, a point of view that was also taken in [1].

For instance, in the nonrelativistic case, two observers with reference frames given by spacetime coordinates $(t, \vec{x})$ and $(T, \vec{X})$ which observe a particle moving with constant speed

$$
\frac{\mathrm{d} \vec{x}(t)}{\mathrm{d} t}=\vec{v}, \quad \frac{\mathrm{d} \vec{X}(T)}{\mathrm{d} T}=\vec{V},
$$

where $\vec{x}(t)$ and $\vec{X}(T)$ are the corresponding trajectories of the particle and $\vec{v}, \vec{V}$ the corresponding constant velocities, or, equivalently,

$$
\frac{\mathrm{d}^{2} \vec{x}(t)}{\mathrm{d} t^{2}}=\overrightarrow{0}, \quad \frac{\mathrm{d}^{2} \vec{X}(T)}{\mathrm{d} T^{2}}=\overrightarrow{0},
$$

are connected by a transformation of the form (2)

$$
\vec{x} \rightarrow \vec{X}=\vec{x}+\delta \vec{x}(t, \vec{x}), \quad t \rightarrow T=t+\delta t(t, \vec{x}),
$$

which, according to the discussion following (2), yields a functional variation

$$
\bar{\delta} \vec{x}=\delta \vec{x}-\dot{\vec{x}} \delta t,
$$

satisfying

$$
\left.\frac{\mathrm{d}^{2} \bar{\delta} \vec{x}(t)}{\mathrm{d} t^{2}}\right|_{\ddot{\vec{x}}=\overrightarrow{0}}=\overrightarrow{0}
$$

This can be repeated for higher order constant motions, such as constant acceleration, constant jerk (time derivative of acceleration), and so on. In the nonrelativistic case, each constant motion is characterized as the zero derivative of the next order, but this is not so straightforward in the relativistic case. It still makes sense, however, to study the symmetries of zero motions in the relativistic case, regardless of whether they can be interpreted as constant motions of the previous order, provided the suitable Lorentz invariant generalizations of jerk, snap, and so on are used. For the jerk this was done in [1], and a generalization to snap and beyond was presented for the first time in [5].

Although a recurrence relation was presented in [5] for these higher order $d+1$ spacetime vectors, it turns out that the study of the symmetries of the corresponding zero motions is better done in terms of a $d$-spatial vector containing the components of the $d+1$ vector, such that the vanishing of the latter is equivalent to the vanishing of the former. These vectors are the nonrelativistic acceleration, nonrelativistic jerk, etc., plus additional terms that guarantee the relativistic transformation properties of the corresponding $d+1$ vectors. This leads to a novel recurrence formula for these spatial vectors appearing in the $(d+1)$-relativistic objects.

The paper is organized as follows. Section II discusses in detail the Lie symmetries of nonrelativistic motions describing constant motions starting with constant position and going up to constant jerk (zero snap), although we also present the general result for arbitrary higher-order constant motions. Section III starts with a summary of the wellknown construction of higher order derivative $d+1$ vectors of the worldline trajectory of a relativistic particle in an arbitrary reference frame [5] and presents new results about the new structure of those vectors. As it was done in the nonrelativistic case, the Lie symmetries of those motions are computed in Sec. IV. Finally, Sec. V summarizes our results. Appendix A proves a lemma used in the main text, and Appendix B discusses some relations in the instantaneous rest frame of a particle. 


\section{LIE SYMMETRIES OF NONRELATIVISTIC MOTIONS}

The Lie symmetries of nonrelativistic motions with constant acceleration (zero jerk) has been studied for a long time. In 1945, Hill [1] studied for the first time the one-dimensional example, in both the relativistic and the nonrelativistic cases. Here we will review the case of $d$ spatial dimensions and then extend the analysis to a constant rate of acceleration (zero snap), and also to the dynamics higher order vanishing derivatives.

\section{A. Nonrelativistic constant position}

We start with the simplest case of constant position, which is somehow special, in that it exhibits an infinite number of point symmetries, and also has a relation with the Carrollian limit of the relativistic case [14].

Consider the equation for nonrelativistic zero velocity in $d$ space dimensions

$$
\frac{\mathrm{d} x^{i}}{\mathrm{~d} t}=0, \quad i=1, \ldots, d
$$

We write the infinitesimal point transformations as

$$
\begin{gathered}
\delta x^{i}=\xi^{i}(t, x), \\
\delta t=f(t, x) .
\end{gathered}
$$

The functional variation is then given by

$$
\bar{\delta} x^{i}(t)=\xi^{i}(t, x)-\dot{x}^{i} f(t, x) .
$$

The Lie symmetries are given by

$$
0=\left.\frac{\mathrm{d}}{\mathrm{d} t} \bar{\delta} x^{i}\right|_{(11)}=\partial_{t} \xi^{i}
$$

and no condition is obtained for $f(t, x)$. The total variation of $x^{i}$ is thus given by an arbitrary space diffeomorphism $\xi^{i}=\xi^{i}(x)$, while $t$ can be transformed by an arbitrary, space-dependent diffeomorphism $\delta t=f(t, x)$. This makes physical sense: space points can be mapped to arbitrary space points and provided the map does not depend on time one still gets a fixed point, while time can arbitrarily be transformed at each point of space. This infinite set of symmetries appears in the study of the symmetries of a Carroll particle [14].

\section{B. Nonrelativistic constant velocity}

The equation of a nonrelativistic particle with a constant velocity in dimensions is

$$
\frac{\mathrm{d}^{2} x^{i}}{\mathrm{~d} t^{2}}=0, \quad i=1, \ldots, d .
$$

We now consider the point transformations (12). The condition that they are Lie symmetries is given by

$$
\begin{aligned}
0= & \left.\frac{\mathrm{d}^{2}}{\mathrm{~d} t^{2}} \bar{\delta} x^{i}\right|_{(16)} \\
= & \partial_{t}^{2} \xi^{i}+2 \dot{x}^{j} \partial_{t} \partial_{j} \xi^{i}+\dot{x}^{k} \dot{x}^{j} \partial_{j} \partial_{k} \xi^{i} \\
& -\dot{x}^{i} \partial_{t}^{2} f-2 \dot{x}^{i} \dot{x}^{j} \partial_{t} \partial_{j} f-\dot{x}^{i} \dot{x}^{k} \dot{x}^{j} \partial_{j} \partial_{k} f .
\end{aligned}
$$

This implies the set of independent equations

$$
\begin{aligned}
\partial_{t}^{2} \xi^{i} & =0, \\
2 \partial_{t} \partial_{j} \xi^{i} & =\delta_{j}^{i} \partial_{t}^{2} f, \\
\partial_{j} \partial_{k} \xi^{i} & =\delta_{k}^{i} \partial_{t} \partial_{j} f+\delta_{j}^{i} \partial_{t} \partial_{k} f, \\
\partial_{j} \partial_{k} f & =0 .
\end{aligned}
$$

From (18) and (19) one finds

$$
\xi^{i}=a^{i}(x)+b^{i}(x) t
$$

$$
f=\alpha(t)+\beta_{i}(t) x^{i}
$$

Since Eqs. (19) and (20) are second order in $x^{i}$ and $t$, the solutions are polynomials of second degree in these variables,

$\xi^{i}=a_{0}^{i}+a_{j}^{i} x^{j}+\frac{1}{2}\left[\delta_{j}^{i} \beta_{1 k}+\delta_{k}^{i} \beta_{1 j}\right] x^{k} x^{j}+\left(b_{0}^{i}+\alpha_{2} x^{i}\right) t$,

$f=\alpha_{0}+\alpha_{1} t+\alpha_{2} t^{2}+\left(\beta_{0 i}+\beta_{1 i} t\right) x^{i}$,

where $a^{i}{ }_{j}$ are the elements of a general $d \times d$ dimensional matrix. The number of independent parameters is $(d+1)(d+3)$, which corresponds to the dimension of the projective group in $d+1$ dimensions, $\mathcal{P}_{d+1}$. The projective group consists of all transformations that map straight lines, which are the solutions to (16), to straight lines; these include rotations, translations, and dilatations. The Schrödinger group $S c h_{d+1}$ is a proper subgroup of the projective group, and it is the first element of the Lisfshitz Galilean conformal algebras with dynamical exponent $z=\frac{2}{N}[9,11]$, in this case with $N=1$.

The vector fields which generate the total variations (24) are, for each parameter, 
TABLE I. Commutators of the Lie symmetry vector fields of (16), with $A_{2}=t^{2} \partial_{t}+t x^{j} \partial_{j}$.

\begin{tabular}{|c|c|c|c|c|c|c|c|c|}
\hline & $\partial_{t}$ & $t \partial_{t}$ & $A_{2}$ & $\partial_{j}$ & $t \partial_{j}$ & $x^{k} \partial_{j}$ & $x^{j} \partial_{t}$ & $B_{1}^{j}$ \\
\hline$\partial_{t}$ & 0 & $\partial_{t}$ & $2 t \partial_{t}+x^{j} \partial_{j}$ & 0 & $\partial_{j}$ & 0 & 0 & $x^{j} \partial_{t}$ \\
\hline$t \partial_{t}$ & & 0 & $A_{2}$ & 0 & $t \partial_{j}$ & 0 & $-x^{j} \partial_{t}$ & 0 \\
\hline$A_{2}$ & & & 0 & $-t \partial_{j}$ & 0 & 0 & $-B_{1}^{j}$ & 0 \\
\hline$\partial_{i}$ & & & & 0 & 0 & $\delta_{i}^{k} \partial_{j}$ & $\delta_{i}^{j} \partial_{t}$ & $\delta_{i}^{j} t \partial_{t}+\delta_{i}^{j} x^{k} \partial_{k}+x^{j} \partial_{t}$ \\
\hline$t \partial_{i}$ & & & & & 0 & $\delta_{i}^{k} t \partial_{j}$ & $\delta_{i}^{j} t \partial_{t}-x^{j} \partial_{i}$ & $\delta_{i}^{j} A_{2}$ \\
\hline$x^{l} \partial_{i}$ & & & & & & $\delta_{i}^{k} x^{l} \partial_{j}-\delta_{j}^{l} x^{k} \partial_{i}$ & $\delta_{i}^{j} x^{l} \partial_{t}$ & $\delta_{i}^{j} B_{1}^{l}$ \\
\hline$x^{i} \partial_{t}$ & & & & & & & 0 & 0 \\
\hline$B_{1}^{i}$ & & & & & & & & 0 \\
\hline
\end{tabular}

$\alpha_{1}: t \partial_{t}$,

$\alpha_{2}: t\left(t \partial_{t}+x^{j} \partial_{j}\right) \equiv A_{2}$,

$a_{0}^{i}: \partial_{i}$,

$b_{0}^{i}: t \partial_{i}$,

$$
a_{j}^{i}: x^{j} \partial_{i}
$$

$$
\beta_{0 i}: x^{i} \partial_{t}
$$

$$
\beta_{1 i}: x^{i}\left(t \partial_{t}+x^{j} \partial_{j}\right) \equiv B_{1}^{i},
$$

and they form the closed algebra given in Table I. Notice that by combining (29) and (31) one obtains the generators of Lorentz boost transformations. In fact, as we will see in Sec. IV, the motions corresponding to zero relativistic acceleration are determined by exactly the same equation (16) of the nonrelativistic case. This result is related to the classification of kinematical algebras [15] in the flat case.

\section{Nonrelativistic constant acceleration}

The condition for constant acceleration, that is, zero jerk, is

$$
\frac{\mathrm{d}^{3} x^{i}}{\mathrm{~d} t^{3}}=0, \quad i=1, \ldots, d
$$

The symmetry conditions are now

$$
\left.\frac{\mathrm{d}^{3}}{\mathrm{~d} t^{3}} \bar{\delta} x^{i}\right|_{(33)}=0
$$

In contrast to the previous case, now $f$ cannot depend on the spatial coordinates $x$. Indeed, since $\bar{\delta} x^{i}=\xi^{i}-\dot{x}^{i} f$, there is a unique term containing quadratic contributions from the accelerations, given by $-3 \ddot{x}^{i} \ddot{x}^{k} \partial_{k} f$, when computing the left-hand side of (34). This implies and using this information the remaining terms yield the independent conditions

$$
\begin{aligned}
\partial_{j} \partial_{k} \xi^{i} & =0, \\
\partial_{t}^{3} \xi^{i} & =0, \\
3 \partial_{t}^{2} \partial_{k} \xi^{i}-\delta^{i}{ }_{k} \partial_{t}^{3} f & =0, \\
\partial_{t} \partial_{k} \xi^{i}-\delta^{i}{ }_{k} \partial_{t}^{2} f & =0 .
\end{aligned}
$$

Combining these equations leads then to

$$
\begin{aligned}
& f=\alpha_{0}+\alpha_{1} t+\alpha_{2} t^{2}, \\
& \xi^{i}=a^{i}+a_{j}^{i} x^{j}+\left(b^{i}+2 \alpha_{2} x^{i}\right) t+c^{i} t^{2} .
\end{aligned}
$$

The associated vector fields are

$$
\begin{aligned}
& \alpha_{0}: \partial_{t}, \\
& \alpha_{1}: t \partial_{t}, \\
& \alpha_{2}: t\left(t \partial_{t}+2 x^{j} \partial_{j}\right) \equiv A_{3}, \\
& a^{i}: \partial_{i}, \\
& b^{i}: t \partial_{i}, \\
& a_{j}^{i}: x^{j} \partial_{i}, \\
& c^{i}: t^{2} \partial_{i},
\end{aligned}
$$

and their commutators form the algebra given in Table II. It has $d^{2}+3 d+3$ generators, and it contains the $N=2$ NGCA algebra without central extension, ${ }^{1}$ with $d(d+1) / 2$

\footnotetext{
${ }^{1}$ A dynamical realization of this algebra for $N=2$ with a central extension was considered in [16] for $d=2$ and for general $d$ in [17], while the extension to arbitrary $N$ was presented in $[10,18]$.
} 
TABLE II. Commutators of the Lie symmetry vector fields of (33), with $A_{3}=t^{2} \partial_{t}+2 t x^{j} \partial_{j}$.

\begin{tabular}{|c|c|c|c|c|c|c|}
\hline & $\partial_{t} t \partial_{t}$ & $A_{3}$ & $\partial_{j}$ & $t \partial_{j}$ & $x^{k} \partial_{j}$ & $t^{2} \partial_{j}$ \\
\hline$\partial_{t}$ & $0 \partial_{t}$ & $2 t \partial_{t}+2 x^{j} \partial_{j}$ & 0 & $\partial_{j}$ & 0 & $2 t \partial_{j}$ \\
\hline$t \partial_{t}$ & 0 & $A_{3}$ & 0 & $t \partial_{j}$ & 0 & $2 t^{2} \partial_{j}$ \\
\hline$A_{3}$ & & 0 & $-2 t \partial_{j}$ & $-t^{2} \partial_{j}$ & 0 & 0 \\
\hline$\partial_{i}$ & & & 0 & 0 & $\delta_{i}^{k} \partial_{j}$ & 0 \\
\hline$t \partial_{i}$ & & & & 0 & $\delta_{i}^{k} t \partial_{j}$ & 0 \\
\hline$x^{l} \partial_{i}$ & & & & & $\delta_{i}^{k} x^{l} \partial_{j}-\delta_{j}^{l} x^{k} \partial_{i}$ & $-\delta_{j}^{l} t^{2} \partial_{i}$ \\
\hline$t^{2} \partial_{i}$ & & & & & & 0 \\
\hline
\end{tabular}

extra generators corresponding to symmetric spatial linear transformations. In contrast to the previous case of zero acceleration, now one cannot construct Lorentz boosts out of these generators, since there is no generator of the form $x^{i} \partial_{t}$. The relativistic counterpart of (33), that is, the equation stating that the $d+1$ relativistic jerk is zero, has additional terms that restore Lorentz invariance of the corresponding $d+1$ vector, at the price of destroying most of the symmetries of (33).

\section{Nonrelativistic constant rate of change of acceleration and beyond}

The equations of motion for a constant rate of acceleration, that is, constant jerk (or zero snap), are

$$
\frac{\mathrm{d}^{4} x^{i}}{\mathrm{~d} t^{4}}=0, \quad i=1, \ldots, d .
$$

The point Lie symmetry transformations satisfy

$$
\left.\frac{\mathrm{d}^{4}}{\mathrm{~d} t^{4}} \bar{\delta} x^{i}\right|_{(48)}=0
$$

Applying the same procedure as in the previous cases one finds

$$
\begin{aligned}
& f=\alpha_{0}+\alpha_{1} t+\alpha_{2} t^{2}, \\
& \xi^{i}=a^{i}+a_{j}^{i} x^{j}+\left(b^{i}+3 \alpha_{2} x^{i}\right) t+c^{i} t^{2}+d^{i} t^{3},
\end{aligned}
$$

which contains $d^{2}+4 d+3$ parameters. The vector fields associated with each parameter are $\partial_{t}\left(\alpha_{0}\right), t \partial_{t}\left(\alpha_{1}\right), A_{4} \equiv$ $t^{2} \partial_{t}+3 t x^{i} \partial_{i}\left(\alpha_{2}\right), \partial_{i}\left(a^{i}\right), x^{j} \partial_{i}\left(a_{j}^{i}\right), t \partial_{i}\left(b^{i}\right), t^{2} \partial_{i}\left(c^{i}\right)$, and $t^{3} \partial_{i}\left(d^{i}\right)$, and they form the closed algebra given in Table III. This algebra contains the $N=3$ NGCA without a central extension, but with $d(d+1) / 2$ extra generators which, again, correspond to arbitrary symmetric spatial linear transformations.

The symmetry transformations corresponding to higher order zero dynamics,

$$
\frac{\mathrm{d}^{n} x^{i}}{\mathrm{~d} t^{n}}=0, \quad i=1, \ldots, d, \quad n \geq 5,
$$

can also be computed, and one obtains

$f=\alpha_{0}+\alpha_{1} t+\alpha_{2} t^{2}$

$\xi^{i}=a^{i}+a_{j}^{i} x^{j}+\left(b^{i}+(N-1) \alpha_{2} x^{i}\right) t+\sum_{k=2}^{n-1} c_{k}^{i} t^{k}$.

This contains the $N=n-1$ NGCA algebra without central extension, again with the extra $d(d+1) / 2$ generators. This also encompasses the cases $n=3$ and $n=4$, but not $n=2$, which is a transition case from the infinite number of symmetries of the case $n=1$ and the regular case for $n \geq 3$. The number of symmetries is $d^{2}+4 d+3$ for $n=2$ and $d^{2}+n d+3$ for $n \geq 3$.

\section{A NEW LOOK AT HIGHER ORDER RELATIVISTIC KINEMATICS}

Let us consider a $d+1$ Minkowski spacetime. We denote by $\tau$ the proper time, $t$ is the lab time, and the corresponding derivatives are denoted by dots and primes, respectively. From the worldline $x^{\mu}(\tau)$ of a particle we

\begin{tabular}{|c|c|c|c|c|c|c|c|c|}
\hline & $\partial_{t}$ & $t \partial_{t}$ & $A_{4}$ & $\partial_{j}$ & $t \partial_{j}$ & $x^{k} \partial_{j}$ & $t^{2} \partial_{j}$ & $t^{3} \partial_{j}$ \\
\hline$\partial_{t}$ & 0 & $\partial_{t}$ & $2 t \partial_{t}+3 x^{j} \partial_{j}$ & 0 & $\partial_{j}$ & 0 & $2 t \partial_{j}$ & $3 t^{2} \partial_{j}$ \\
\hline$t \partial_{t}$ & & 0 & $A_{3}$ & 0 & $t \partial_{j}$ & 0 & $2 t^{2} \partial_{j}$ & $3 t^{3} \partial_{j}$ \\
\hline$A_{4}$ & & & 0 & $-3 t \partial_{j}$ & $-2 t^{2} \partial_{j}$ & 0 & $-t^{3} \partial_{j}$ & 0 \\
\hline$\partial_{i}$ & & & & 0 & 0 & $\delta_{j}^{k} \partial_{j}$ & 0 & 0 \\
\hline$t \partial_{i}$ & & & & & 0 & $\delta_{i}^{k} t \partial_{j}$ & 0 & 0 \\
\hline$x^{l} \partial_{i}$ & & & & & & $\delta_{i}^{k} x^{l} \partial_{j}-\delta_{j}^{l} x^{k} \partial_{i}$ & $-\delta_{j}^{l} t^{2} \partial_{i}$ & $-\delta_{j}^{l} t^{3} \partial_{i}$ \\
\hline$t^{2} \partial_{i}$ & & & & & & & 0 & 0 \\
\hline$t^{3} \partial_{i}$ & & & & & & & & 0 \\
\hline
\end{tabular}
construct

$v^{\mu}=\dot{x}^{\mu}, \quad a^{\mu}=\dot{v}^{\mu}, \quad b^{\mu}=\dot{a}^{\mu}, \quad h^{\mu}=\dot{b}^{\mu}$.

TABLE III. Commutators of the Lie symmetry vector fields of (48), with $A_{4}=\equiv t^{2} \partial_{t}+3 t x^{i} \partial_{i}$. 
In the lab frame one has

$$
x^{\mu}=(c t, \vec{x}) .
$$

We define also

$$
\vec{v}=\vec{x}^{\prime}, \quad \vec{a}=\vec{v}^{\prime}, \quad \vec{b}=\vec{a}^{\prime}, \quad \vec{h}=\vec{b}^{\prime} .
$$

Taking into account that

$$
\frac{\mathrm{d}}{\mathrm{d} t}=\frac{1}{\gamma} \frac{\mathrm{d}}{\mathrm{d} \tau}, \quad \gamma=\left(1-\frac{\vec{v}^{2}}{c^{2}}\right)^{-1 / 2},
$$

one gets

$$
\begin{aligned}
v^{\mu}= & (\gamma c, \gamma \vec{v}), \\
a^{\mu}= & \left(\gamma^{4} \frac{\vec{v} \cdot \vec{a}}{c}, \gamma^{4} \frac{\vec{v} \cdot \vec{a}}{c^{2}} \vec{v}+\gamma^{2} \vec{a}\right), \\
b^{\mu}= & \left(4 \gamma^{7} \frac{(\vec{v} \cdot \vec{a})^{2}}{c^{3}}+\frac{\gamma^{5}}{c}\left(\vec{a}^{2}+\vec{v} \cdot \vec{b}\right), \gamma^{3} \vec{b}+4 \gamma^{7} \frac{(\vec{v} \cdot \vec{a})^{2}}{c^{4}} \vec{v}\right. \\
& \left.+\frac{\gamma^{5}}{c^{2}}\left(\vec{a}^{2}+\vec{v} \cdot \vec{b}\right) \vec{v}+3 \gamma^{5} \frac{\vec{v} \cdot \vec{a}}{c^{2}} \vec{a}\right),
\end{aligned}
$$

where we have also used

$$
\gamma^{\prime}=\gamma^{3} \frac{\vec{v} \cdot \vec{a}}{c^{2}} .
$$

From the expression of $v^{\mu}$ one has

$$
v^{\mu} v_{\mu}=-c^{2},
$$

and then, deriving with respect to $\tau$,

$$
\begin{aligned}
v^{\mu} a_{\mu} & =0, \\
a^{\mu} a_{\mu}+v^{\mu} b_{\mu} & =0, \\
3 a^{\mu} b_{\mu}+v^{\mu} h_{\mu} & =0 .
\end{aligned}
$$

Note, in particular, that neither $b^{\mu}$ nor $h^{\mu}$ are orthogonal to the velocity. Following [5], we define the relativistic jerk $j^{\mu}$ as the component of $b^{\mu}$ orthogonal to $v^{\mu}$,

$$
j^{\mu}=b^{\mu}-\frac{b^{\nu} v_{\nu}}{v^{2}} v^{\mu}=b^{\mu}+\frac{b^{\nu} v_{\nu}}{c^{2}} v^{\mu} .
$$

After some algebra one finds out that the temporal part of $j^{\mu}$ is given by

$$
j^{0}=3 \gamma^{7} \frac{(\vec{v} \cdot \vec{a})^{2}}{c^{3}}+\gamma^{5} \frac{\vec{v} \cdot \vec{b}}{c},
$$

while the spatial components are ${ }^{2}$

$j^{i}=\gamma^{3} b^{i}+3 \gamma^{5} \frac{\vec{v} \cdot \vec{a}}{c^{2}} a^{i}+3 \gamma^{7} \frac{(\vec{v} \cdot \vec{a})^{2}}{c^{4}} v^{i}+\gamma^{5} \frac{\vec{v} \cdot \vec{b}}{c^{2}} v^{i}$.

We define the relativistic snap, $s^{\mu}$, as the derivative of $j^{\mu}$ (instead of $b^{\mu}$ ) with respect to $\tau$ and subtract again the component along $v^{\mu}$ :

$$
s^{\mu}=\frac{\mathrm{d} j^{\mu}}{\mathrm{d} \tau}-\frac{\frac{\mathrm{d} j^{\nu}}{\mathrm{d} \tau} v_{\nu}}{v^{2}} v^{\mu}=\frac{\mathrm{d} j^{\mu}}{\mathrm{d} \tau}+\frac{\frac{\mathrm{d} j^{\nu}}{\mathrm{d} \tau} v_{\nu}}{c^{2}} v^{\mu} .
$$

After some algebra this yields

$$
s^{\mu}=h^{\mu}-\frac{a^{2}}{c^{2}} a^{\mu}-3 \frac{a^{\nu} b_{\nu}}{c^{2}} v^{\mu} .
$$

The four vectors $a^{\mu}, j^{\mu}, s^{\mu}$, whose explicit expressions were given in [5], are such that they have purely spatial components in the comoving frame, $v^{\mu}=0$, and can be rewritten in compact form as

$$
\begin{aligned}
a^{\mu} & =\left(\frac{\gamma^{4}}{c} \vec{v} \cdot \vec{A}, \gamma^{2} \hat{M} \vec{A}\right), \\
j^{\mu} & =\left(\frac{\gamma^{5}}{c} \vec{v} \cdot \vec{B}, \gamma^{3} \hat{M} \vec{B}\right), \\
s^{\mu} & =\left(\frac{\gamma^{6}}{c} \vec{v} \cdot \vec{H}, \gamma^{4} \hat{M} \vec{H}\right) .
\end{aligned}
$$

Here we have defined

$$
\begin{aligned}
\vec{A}= & \vec{a}, \\
\vec{B}= & \vec{b}+3 \gamma^{2} \frac{\vec{v} \cdot \vec{a}}{c^{2}} \vec{a}, \\
\vec{H}= & \vec{h}+6 \gamma^{2} \frac{\vec{v} \cdot \vec{a}}{c^{2}} \vec{b} \\
& +\left(3 \gamma^{2} \frac{\vec{a}^{2}}{c^{2}}+4 \gamma^{2} \frac{\vec{v} \cdot \vec{b}}{c^{2}}+18 \gamma^{4} \frac{(\vec{v} \cdot \vec{a})^{2}}{c^{4}}\right) \vec{a} \\
= & \vec{B}^{\prime}+3 \gamma^{2} \frac{\vec{v} \cdot \vec{a}}{c^{2}} \vec{B}+\gamma^{2} \frac{\vec{v} \cdot \vec{B}}{c^{2}} \vec{a},
\end{aligned}
$$

where the matrix $\hat{M}$ has components

$$
\hat{M}_{i j}=\delta_{i j}+\frac{\gamma^{2}}{c^{2}} v_{i} v_{j}
$$

\footnotetext{
${ }^{2} v^{i}, a^{i}, b^{i}$ denote the components of the Euclidean vectors $\vec{v}, \vec{a}$, $\vec{b}$, respectively, not the spatial components of $v^{\mu}, a^{\mu}, b^{\mu}$.
} 
whose inverse is given by

$$
\hat{M}_{i j}^{-1}=\delta_{i j}-\frac{1}{c^{2}} v_{i} v_{j}
$$

The appearance of the spatial vectors $\vec{A}, \vec{B}, \vec{C}$ in the components of the corresponding $(d+1)$-vectors $a^{\mu}, j^{\mu}, s^{\mu}$, together with the invertibility of $\hat{M}$, has interesting consequences. In particular, the vanishing of the relativistic acceleration, jerk, snap, ..., is equivalent, in each case, to the vanishing of the corresponding $d$-dimensional spatial vectors. These vectors are the $d$-dimensional nonrelativistic ones plus additional terms that guarantee the relativistic transformation properties of the corresponding $(d+1)$ dimensional vectors. Explicitly,

$$
\begin{gathered}
a^{\mu}=0 \Leftrightarrow \vec{A}=0, \\
j^{\mu}=0 \Leftrightarrow \vec{B}=0, \\
s^{\mu}=0 \Leftrightarrow \vec{H}=0 .
\end{gathered}
$$

From these expressions it also follows that

$$
\begin{aligned}
\left.\vec{B}\right|_{\vec{A}=0} & =0, \\
\left.\vec{H}\right|_{\vec{B}=0} & =0 .
\end{aligned}
$$

As shown in [5], the sequence of $(d+1)$-vectors $a^{\mu}, j^{\mu}$, $s^{\mu}$ can be extended to higher orders by means of the recurrence relation

$$
P_{(n)}^{\mu}=\frac{\mathrm{d}}{\mathrm{d} \tau} P_{(n-1)}^{\mu}-\frac{P_{(n-1)}^{\nu} a_{\nu}}{c^{2}} v^{\mu},
$$

starting with $P_{(1)}^{\mu}=a^{\mu}$. This recurrence relation preserves the property of orthogonality with $v$, since

$$
v_{\mu} P_{(n)}^{\mu}=\frac{\mathrm{d}}{\mathrm{d} \tau}\left(v_{\mu} P_{(n-1)}^{\mu}\right)=0
$$

provided $v_{\mu} P_{(n-1)}^{\mu}=0$. Using

$$
v_{\nu} \frac{\mathrm{d}}{\mathrm{d} \tau} P_{(n-1)}^{\nu}=-a_{\nu} P_{(n-1)}^{\nu},
$$

Eq. (86) can be rewritten as

$$
P_{(n)}^{\mu}=\left(\delta_{\nu}^{\mu}+\frac{v_{\nu} v^{\mu}}{c^{2}}\right) \frac{\mathrm{d}}{\mathrm{d} \tau} P_{(n-1)}^{\mu}
$$

This allows for an interesting generalization of (72), (73), (74) that provides, in addition, a recurrence relation for the objects $\vec{A}, \vec{B}, \vec{H}$,

Proposition 1: The $(d+1)$-vectors $P_{(n)}^{\mu}$ defined by (89), starting with $P_{(2)}^{\mu}=a^{\mu}$, can be written as

$$
P_{(n)}^{\mu}=\left(\frac{\gamma^{n+3}}{c} \vec{v} \cdot \vec{Q}_{(n)}, \gamma^{n+1} \hat{M} \vec{Q}_{(n)}\right), \quad n \geq 2,
$$

with $\hat{M}$ the $d \times d$ matrix given in (79), and with the $d$ vectors $\vec{Q}_{(n)}$ satisfying the recurrence relation

$\vec{Q}_{(n)}=\frac{1}{\gamma^{n}} \frac{\mathrm{d}}{\mathrm{d} t}\left(\gamma^{n} \vec{Q}_{(n-1)}\right)+\frac{\gamma^{2}}{c^{2}}\left(\vec{v} \cdot \vec{Q}_{(n-1)}\right) \vec{a}, \quad n \geq 3$,

starting with $\vec{Q}_{(2)}=\vec{A}$.

In order to prove this result we need the following lemma, which is proved in Appendix A.

\section{Lemma 1: Let}

$$
Y^{\mu}=\left(\delta^{\mu}{ }_{\nu}+\frac{v_{\nu} v^{\mu}}{c^{2}}\right) X^{\mu}
$$

Then $Y^{\mu}$ can be written as

$$
Y^{\mu}=\left(\frac{\gamma^{2}}{c} \vec{v} \cdot \vec{K}, \hat{M} \vec{K}\right)
$$

with

$$
\vec{K}=\vec{Y}-\frac{Y^{0}}{c} \vec{v}
$$

Furthermore,

$$
Y^{2}=\vec{K}^{2}+\frac{\gamma^{2}}{c^{2}}(\vec{v} \cdot \vec{K})^{2} \geq 0,
$$

or, equivalently,

$$
v \cdot Y=0 .
$$

Proof of Proposition 1: From (89) it follows that $P_{(n)}^{\mu}$ obeys a relation of the type given in (92), with $X^{\mu}=$ $\frac{\mathrm{d}}{\mathrm{d} \tau} P_{(n-1)}^{\mu}$ for each $n$, and hence there exists a $\vec{K}_{(n)}$ such that it can be expressed as in (93) and (94). In particular, 


$$
\begin{aligned}
K_{(n)}^{i} & =P_{(n)}^{i}-\frac{v^{i}}{c} P_{(n)}^{0} \\
& =\frac{\mathrm{d}}{\mathrm{d} \tau} P_{(n-1)}^{i}-\frac{\gamma}{c^{2}} P_{(n-1)}^{\nu} a_{\nu} v^{i}-\frac{v^{i}}{c}\left(\frac{\mathrm{d}}{\mathrm{d} \tau} P_{(n-1)}^{0}-\frac{\gamma}{c} P_{(n-1)}^{\nu} a_{\nu}\right) \\
& =\frac{\mathrm{d}}{\mathrm{d} \tau}\left(P_{(n-1)}^{i}-\frac{v^{i}}{c} P_{(n-1)}^{0}\right)+\frac{1}{c} P_{(n-1)}^{0} \frac{\mathrm{d} v^{i}}{\mathrm{~d} \tau} \\
& =\frac{\mathrm{d}}{\mathrm{d} \tau} K_{(n-1)}^{i}+\frac{1}{c} P_{(n-1)}^{0} \frac{\mathrm{d} v^{i}}{\mathrm{~d} \tau} \\
& =\gamma \frac{\mathrm{d}}{\mathrm{d} t} K_{(n-1)}^{i}+\frac{\gamma \mathrm{d} v^{i} \gamma^{2}}{\mathrm{~d} t} \frac{\vec{c}}{c}\left(\overrightarrow{\left.K_{(n-1)}\right)}\right. \\
& =\gamma \frac{\mathrm{d}}{\mathrm{d} t} K_{(n-1)}^{i}+\frac{\gamma^{3}}{c^{2}}\left(\vec{v} \cdot \vec{K}_{(n-1)}\right) a^{i} .
\end{aligned}
$$

Introducing now $Q_{(n)}^{i}$ by means of

$$
K_{(n)}^{i}=\gamma^{n+1} Q_{(n)}^{i},
$$

Eq. (97) for the $\vec{K}$ 's can be rewritten as

$$
\gamma^{n+1} Q_{(n)}^{i}=\gamma \frac{\mathrm{d}}{\mathrm{d} t}\left(\gamma^{n} Q_{(n-1)}^{i}\right)+\frac{\gamma^{n+3}}{c^{2}}\left(\vec{v} \cdot \vec{Q}_{(n-1)}\right) a^{i},
$$

which, after some algebra, becomes (91). Finally, using (93) for $P_{(n)}^{\mu}$ and expressing $\vec{K}_{(n)}$ in terms of $\vec{Q}_{(n)}$ yields (90), and this concludes the proof.

Using (90) and (91), the next term in the sequence $a^{\mu}, b^{\mu}$, $j^{\mu}, s^{\mu}$ would be the relativistic "crackle" $k^{\mu}$, given by

$$
k^{\mu}=P_{(4)}^{\mu}=\left(\frac{\gamma^{7}}{c} \vec{v} \cdot \vec{C}, \gamma^{5} \hat{M} \vec{C}\right),
$$

with the spatial vector $\vec{C}$ obtained from $\vec{H}$ by

$$
\begin{aligned}
\vec{C} & =\frac{1}{\gamma^{4}} \frac{\mathrm{d}}{\mathrm{d} t}\left(\gamma^{4} \vec{H}\right)+\frac{\gamma^{2}}{c^{2}}(\vec{v} \cdot \vec{H}) \vec{a} \\
& =\frac{\mathrm{d} \vec{H}}{\mathrm{~d} t}+\frac{4}{\gamma} \frac{\mathrm{d} \gamma}{\mathrm{d} t} \vec{H}+\frac{\gamma^{2}}{c^{2}}(\vec{v} \cdot \vec{H}) \vec{a} .
\end{aligned}
$$

As in the lower order cases, Eq. (91) shows that if $\vec{Q}_{(n)}$ vanishes, then $\vec{Q}_{(m)}$ is also identically zero for all $m>n$; furthermore, from (90) the invertibility of $\hat{M}$ implies that $P_{(n)}=0$ if and only if $\vec{Q}_{(n)}=0$. On the other hand, one can also see from (91) —or from (86) for that matter-that $\vec{Q}_{(n)}=\overrightarrow{0}$ does not necessarily imply that $\vec{Q}_{(n-1)}$ is constant, marking a departure from the situation in the nonrelativistic case. In fact, the question of defining movements with constant jerk, snap, and beyond is conceptually complex $[5,6]$. We will just present a short discussion, in the $1+1$ spacetime case in Appendix B, using the $d$-dimensional spatial vectors we have encountered.

\section{LIE SYMMETRIES OF HIGHER RELATIVISTIC DYNAMICS}

In this section we will compute the Lie symmetries of the equations of motion corresponding to zero relativistic acceleration, jerk, snap, and beyond in an arbitrary lab frame. As commented in the Introduction, and following the ideas in [1], these symmetries can be seen as the spacetime transformations that connect different lab frames where the corresponding zero motion is preserved.

\section{A. Symmetries of $\vec{A}=0$}

Since the relativistic zero acceleration is finally equivalent to $\vec{A}=0$, one has the equations

$$
\frac{\mathrm{d}^{2} x^{i}}{\mathrm{~d} t^{2}}=0
$$

which are the same as those of the nonrelativistic zero acceleration case. This means that the set of Lie symmetries is given by the fields (25)-(32). This is not the case for higher order zero motions, for which the relevant quantities exhibit extra terms [see (76) and (77)].

\section{B. Symmetries of $\vec{B}=0$}

The equations of motion $B_{i}=0$ are

$$
b_{i}+3 \frac{v_{k} a_{k}}{c^{2}-v_{k}^{2}} a_{i}=0, \quad i=1, \ldots, d .
$$

Proceeding as in the nonrelativistic case, one finds out that the symmetries of (103) are given by the $d(d-1) / 2+3 d+3$ vector fields

$$
\begin{aligned}
& \partial_{t}, \\
& \partial_{i}, \\
& B_{i} \equiv M_{0 i}=x_{i} \partial_{t}+c^{2} t \partial_{i}, \\
& M_{i j}=x_{j} \partial_{i}-x_{i} \partial_{j}, \\
& D=t \partial_{t}+x^{i} \partial_{i}, \\
& C_{i}=\left(c^{2} t^{2}-x^{j} x_{j}\right) \partial_{i}+2 x_{i}\left(t \partial_{t}+x^{j} \partial_{j}\right), \\
& C=\left(c^{2} t^{2}+x^{j} x_{j}\right) \partial_{t}+2 c^{2} t x^{j} \partial_{j},
\end{aligned}
$$

whose commutators, given in Table IV, form the conformal algebra in $d+1$ Minkowski spacetime. This result generalizes that of [1] in the one-dimensional case.

\section{Symmetries of $\overrightarrow{\boldsymbol{H}}=0$}

Finally, the symmetries of the fourth order differential equations 
TABLE IV. Commutators of the Lie symmetry vector fields of (103).

\begin{tabular}{lccccccc}
\hline \hline & $\partial_{t}$ & $\partial_{j}$ & $B_{j}$ & $M_{j k}$ & $D$ & $C_{j}$ & $C$ \\
\hline$\partial_{t}$ & 0 & 0 & $c^{2} \partial_{j}$ & 0 & $\partial_{t}$ & $2 B_{j}$ & $2 c^{2} D$ \\
$\partial_{i}$ & & 0 & $\delta_{i j} \partial_{t}$ & $\delta_{i j} \partial_{k}-\delta_{i k} \partial_{j}$ & $\partial_{i}$ & $2 M_{i j}+2 \delta_{i j} D$ & $2 B_{i}$ \\
$B_{i}$ & & & $-c^{2} M_{i j}$ & $\delta_{i j} B_{k}-\delta_{i k} B_{j}$ & 0 & $\delta_{i j} C$ & $c^{2} C_{i}$ \\
$M_{i l}$ & & & & $-\delta_{l j} M_{i k}+\delta_{l k} M_{i j}+\delta_{i j} M_{l k}-\delta_{i k} M_{l j}$ & 0 & $\delta_{i j} C_{l}-\delta_{l j} C_{i}$ & 0 \\
$D$ & & & & & 0 & $C_{j}$ & $C$ \\
$C_{i}$ & & & & & & 0 \\
$C$ & & & & & & 0 \\
\hline \hline
\end{tabular}

$$
H^{i}=0, \quad i=1, \ldots, d,
$$

are described by the $d(d-1) / 2+2 d+2$ vector fields

$$
\begin{gathered}
\partial_{t}, \\
\partial_{i}, \\
B_{i}=x_{i} \partial_{t}+c^{2} t \partial_{i}, \\
M_{i j}=x_{j} \partial_{i}-x_{i} \partial_{j}, \\
D=t \partial_{t}+x^{i} \partial_{i},
\end{gathered}
$$

whose commutators satisfy the Weyl algebra (the subalgebra of the conformal algebra in $d+1$ given in the $5 \times 5$ upper-left part of Table IV).

\section{Symmetries of higher order zero motions}

The symmetries of higher order zero motions,

$$
P_{(n)}^{\mu}=0, \quad n \geq 5
$$

which, according to (90), are those of

$$
Q_{(n)}^{i}=0, \quad i=1, \ldots, d, \quad n \geq 5,
$$

already appearing for $n=4$, i.e., in the snap case. Thus, the group of symmetries remains the Weyl group for all higher order zero motions. This is in contrast with the nonrelativistic case, where the algebra of symmetries grows due to the enlargement of the NGCA algebra with $N$.

\section{CONCLUSIONS}

Table V summarizes our results with respect to symmetry algebras of the zero dynamics that we have studied in this paper. We have also considered $n=1$ in the nonrelativistic case, which yields an infinite dimensional algebra and which is not shown in the table.

Beyond $n=2$, the number of symmetries of zero motions in the nonrelativistic case increases with $n$ because the number of generators in the $N$ Galilean conformal algebra increases with $N$. In contrast to this, in the relativistic case the number of symmetries decreases with $n$, and this answers in the negative our initial question about the existence of a family of higher order relativistic conformal algebras. Conformal algebras only appear in the relativistic case for $n=2$ and $n=3$, the latter being the standard conformal algebra, and from this point onwards there is only a vestige of conformal symmetry in the form of spacetime dilatations.

For $n=2$ the set of Lie symmetries are the same for the relativistic and nonrelativistc cases. For $n=1$ only the nonrelativistic case can be considered, since the relativistic velocity $v^{\mu}=(\gamma c, \gamma \vec{v})$ cannot be made equal to zero unless we take $c=0$. In fact, in the nonrelativistic case, zero velocity motions, that is, constant position motions, have the same Lie symmetries as those of the Carrollian limit $(c \rightarrow 0)$ of a relativistic particle.

Although we have limited ourselves to Lie symmetries of the zero motions, one can also study the Noether symmetries of the actions which, when available, yield the corresponding equations of motion. In general, the Noether symmetries form a subgroup of the Lie ones. Such actions are easily available for nonrelativistic even order zero motions, as is also the case for the zero acceleration relativistic movement; its existence and interpretation for

TABLE V. Algebra of Lie symmetries corresponding to several nonrelativistic and relativistic zero dynamics in $d+1$ spacetime. The superscript $*$ indicates $d(d+1) / 2$ extra generators added to those of the standard NGCA.

\begin{tabular}{lll}
\hline \hline$n$-order zero dynamics & \multicolumn{1}{c}{ Nonrelativistic } & Relativistic \\
\hline$n=2$ & Projective in $d+1$ (contains $N=1$ NGCA) & Projective in $d+1$ \\
$n=3$ & Extended* NGCA $N=2$ in $d+1$ & Conformal in $d+1$ \\
$n \geq 4$ & Extended* NGCA $N=n-1$ in $d+1$ & Weyl in $d+1$ \\
\hline \hline
\end{tabular}


odd order in the nonrelativistic case and in the general relativistic one is under study. Since the symmetries presented here are more general than those of the standard NGCA it would also be interesting to use the technique of nonlinear realizations (see, for instance, [16-19]) to construct the corresponding actions.

In the relativistic case one could also consider the Lie symmetries of the equations of motion when these are expressed using the proper time as the independent variable instead of the time in an arbitrary frame, adding the proper time condition as a new equation of motion. For zero acceleration the set of Lie symmetries boils down to the Weyl group, from the full projective group when using the arbitrary frame time. Such a reduction in the symmetry has also been reported, in the context of the equations of motion derived from a Lagrangian without fixing the gauge, in [20].

\section{ACKNOWLEDGMENTS}

We thank Avijit Lahiri, Anindya Ghose Choudhury, G. P. Sastry, Sayan Kar, Koushik Dutta, Maximilian Wentzel, Soumya Ray, Siddhartha Dechoudhury, Axel Kleinschmidt, Jorge Russo, Paul Townsend, and Josep Maria Pons for useful discussions. The work of C. B. has been partially supported by Generalitat de Catalunya through Project No. 2017 SGR 872. J.G. has been supported in part by MINECO FPA2016-76005-C2-1-P and Consolider CPAN, and by the Spanish government (MINECO/FEDER) under Project No. MDM-2014-0369 of ICCUB (Unidad de Excelencia María de Maeztu). J.G. has also been supported by CONICYT under Grant No. PAI801620047 as a visiting professor of the Universidad Austral de Chile. S.R. is supported by FONDECYT Grant No. 1150907. J.Z. has been partially funded by FONDECYT Grant No. 1180368. The Centro de Estudios Científicos (CECs) is funded by the Chilean Government through the Centers of Excellence Base Financing Program of Conicyt.

\section{APPENDIX A: PROOF OF LEMMA 1}

From (92) one has

$$
\begin{aligned}
& Y^{i}=X^{i}+\gamma(X \cdot v) \frac{v^{i}}{c^{2}}, \\
& Y^{0}=X^{0}+\gamma(X \cdot v) \frac{1}{c} .
\end{aligned}
$$

Combining these one gets

$$
Y^{i}-\frac{v^{i}}{c} Y^{0}=X^{i}-\frac{v^{i}}{c} X^{0} \equiv K^{i},
$$

where $K^{i}$ is a constant quantity, the same for all the elements of the sequence of $d+1$ vectors given by (92). From (A2) one now gets

$$
\begin{aligned}
Y^{0} & =X^{0}-\gamma^{2} X^{0}+(\vec{X} \cdot \vec{v}) \frac{\gamma^{2}}{c} \\
& =(\vec{X} \cdot \vec{v}) \frac{\gamma^{2}}{c}-\gamma^{2} \frac{\vec{v}^{2}}{c^{2}} X^{0} \\
& =\frac{\gamma^{2}}{c} \vec{v} \cdot\left(\vec{X}-\frac{X^{0}}{c} \vec{v}\right) \\
& =\frac{\gamma^{2}}{c} \vec{v} \cdot \vec{K},
\end{aligned}
$$

and then

$$
Y^{i}=K^{i}+\frac{v^{i}}{c} Y^{0}=K^{i}+\frac{\gamma^{2}}{c^{2}}(\vec{v} \cdot \vec{K}) v^{i} .
$$

Putting (A3) and (A4) together one has

$$
\begin{aligned}
Y^{\mu} & =\left(\frac{\gamma^{2}}{c} \vec{v} \cdot \vec{K}, \vec{K}+\frac{\gamma^{2}}{c^{2}}(\vec{v} \cdot \vec{K}) \vec{v} .\right) \\
& =\left(\frac{\gamma^{2}}{c} \vec{v} \cdot \vec{K}, \hat{M} \vec{K}\right)
\end{aligned}
$$

with

$$
\hat{M}_{i j}=\delta_{i j}+\frac{\gamma^{2}}{c^{2}} v_{i} v_{j}
$$

as desired. The last two results of the lemma follow then from a simple calculation.

\section{APPENDIX B: INTERPRETATION OF HIGHER ORDER ZERO DYNAMICS IN THE INSTANTANEOUS REST FRAME}

We show here that, at least in $1+1$, requiring $\vec{B}=0$ and $\vec{H}=0$ is equivalent to having constant values for the spatial part of the corresponding quantities of one order less in the instantaneous rest frame of the particle; i.e., $\vec{B}=0$ in the lab frame is equivalent to constant acceleration in the rest frame, and so on. We do not have at present an equivalent result in $d+1$.

In $(1+1)$ dimensions equations (72), (73), (74) boil down to

$$
\begin{aligned}
a^{\mu} & =\left(\frac{\gamma^{4}}{c} v A, \gamma^{4} A\right), \\
j^{\mu} & =\left(\frac{\gamma^{5}}{c} v B, \gamma^{5} B\right), \\
s^{\mu} & =\left(\frac{\gamma^{6}}{c} v H, \gamma^{6} H\right),
\end{aligned}
$$

with 


$$
\begin{aligned}
& A=a, \\
& B=b+3 \frac{\gamma^{2}}{c^{2}} v a^{2}, \\
& H=h+10 \frac{\gamma^{2}}{c^{2}} v a b+3 \frac{\gamma^{2}}{c^{2}} a^{3}+18 \frac{\gamma^{4}}{c^{4}} v^{2} a^{3} .
\end{aligned}
$$

A general Lorentz transformation in $1+1$ is given by

$$
\Lambda(v)=\left(\begin{array}{cc}
\gamma & -\gamma \frac{v}{c} \\
-\gamma \frac{v}{c} & \gamma
\end{array}\right)
$$

and $a^{\mu}, j^{\mu}, s^{\mu}$ in the rest frame are then

$$
\begin{aligned}
a_{\mathrm{co}}^{\mu} & =\Lambda(v) a^{\mu}=\left(\begin{array}{c}
0 \\
\gamma^{3} a
\end{array}\right) \equiv\left(\begin{array}{c}
0 \\
a_{\mathrm{co}}
\end{array}\right), \\
j_{\mathrm{co}}^{\mu} & =\Lambda(v) j^{\mu}=\left(\begin{array}{c}
0 \\
\gamma^{4} b+3 \frac{\gamma^{6}}{c^{2}} v a^{2}
\end{array}\right) \equiv\left(\begin{array}{c}
0 \\
b_{\mathrm{co}}
\end{array}\right), \\
s_{\mathrm{co}}^{\mu} & =\Lambda(v) s^{\mu}=\left(\begin{array}{c}
0 \\
\gamma^{5} h+10 \frac{\gamma^{7}}{c^{2}} v a b+3 \frac{\gamma^{7}}{c^{2}} a^{3}+18 \frac{\gamma^{9}}{c^{4}} v^{2} a^{3}
\end{array}\right) \\
& \equiv\left(\begin{array}{c}
0 \\
h_{\mathrm{co}}
\end{array}\right),
\end{aligned}
$$

where $a_{\mathrm{co}}, b_{\mathrm{co}}, h_{\mathrm{co}}$ represent the spatial part of the $1+1$ acceleration, jerk, and snap, respectively, in the instantaneous rest frame.

A simple calculation shows that

$$
\begin{aligned}
\frac{\mathrm{d}}{\mathrm{d} t}\left(a_{\mathrm{co}}\right) & =\frac{\mathrm{d}}{\mathrm{d} t}\left(\gamma^{3} a\right)=\gamma^{3} b+3 \gamma^{2} \frac{\mathrm{d} \gamma}{\mathrm{d} t} a \\
& =\gamma^{3} b+3 \gamma^{5} \frac{v a}{c^{2}} a=\gamma^{3} B,
\end{aligned}
$$

from which it follows that, in $1+1$, zero lab jerk $(B=0)$ implies that the spatial acceleration measured in the rest frame is constant. Analogously,

$$
\frac{\mathrm{d}}{\mathrm{d} t}\left(\gamma^{4} b+3 \frac{\gamma^{6}}{c^{2}} v a^{2}\right)=\gamma^{4} H
$$

showing that, in $1+1$, zero lab snap $(H=0)$ is equivalent to constant rest jerk.

The $1+1$ Lorentz transformation (B7) can be parametrized as

$$
\Lambda(v)=\left(\begin{array}{cc}
\cosh \phi & -\sinh \phi \\
-\sinh \phi & \cosh \phi
\end{array}\right)
$$

with $\phi(\tau)$ arbitrary. The velocity in the lab frame can be obtained as

$$
v=c \tanh \phi
$$

and the derivative of the lab time with respect to the proper time is then

$$
\frac{\mathrm{d} t}{\mathrm{~d} \tau}=\gamma=\cosh \phi
$$

Deriving $v$ with respect to $t$ and using (B15) to express the derivatives in terms of those of $\phi(\tau)$ with respect to $\tau$ (denoted by primes), one gets

$$
\begin{aligned}
a=\frac{\mathrm{d} v}{\mathrm{~d} t}= & \frac{c}{\cosh ^{3} \phi} \phi^{\prime}, \\
b=\frac{\mathrm{d} a}{\mathrm{~d} t}= & -\frac{3 c \sinh \phi}{\cosh ^{5} \phi}\left(\phi^{\prime}\right)^{2}+\frac{c}{\cosh ^{4} \phi} \phi^{\prime \prime}, \\
h=\frac{\mathrm{d} b}{\mathrm{~d} t}= & -\frac{3 c}{\cosh ^{5} \phi}\left(\phi^{\prime}\right)^{3}+\frac{15 c \sinh ^{2} \phi}{\cosh ^{7} \phi}\left(\phi^{\prime}\right)^{3} \\
& -\frac{10 c \sinh ^{3} \phi}{\cosh ^{6} \phi} \phi^{\prime} \phi^{\prime \prime}+\frac{c}{\cosh ^{5} \phi} \phi^{\prime \prime \prime},
\end{aligned}
$$

and so on. Then,

(1) if $\phi(\tau)=\frac{v_{0}}{c}$, then $a=b=h=\cdots=0$.

(2) if $\phi(\tau)=\frac{1}{c}\left(v_{0}+a_{0} \tau\right)$, then

$$
\begin{aligned}
& a=\frac{1}{\gamma^{3}} a_{0}, \\
& b=-\frac{3 v}{\gamma^{4}} \frac{a_{0}^{2}}{c^{2}} \neq 0, \\
& h=\frac{3\left(4 \gamma^{2}-5\right)}{c^{2} \gamma^{7}} a_{0}^{3} \neq 0,
\end{aligned}
$$

but it follows from these equations that, in the rest frame,

$$
\begin{aligned}
a_{\mathrm{co}}= & \gamma^{3} a=a_{0}, \\
b_{\mathrm{co}}= & \gamma^{4} b+3 \frac{\gamma^{6}}{c^{2}} v a^{2}=0, \\
h_{\mathrm{co}}= & \gamma^{5} h+10 \frac{\gamma^{7}}{c^{2}} v a b \\
& +3 \frac{\gamma^{7}}{c^{2}} a^{3}+18 \frac{\gamma^{9}}{c^{4}} v^{2} a^{3}=0 .
\end{aligned}
$$


(3) if $\phi(\tau)=\frac{1}{c}\left(v_{0}+a_{0} \tau+\frac{1}{2} j_{0} \tau^{2}\right)$ one gets, in the rest frame of the particle,

$$
\begin{aligned}
a_{\mathrm{co}}= & \gamma^{3} a=a_{0}+j_{0} \tau, \\
b_{\mathrm{co}}= & \gamma^{4} b+3 \frac{\gamma^{6}}{c^{2}} v a^{2}=j_{0}, \\
h_{\mathrm{co}}= & \gamma^{5} h+10 \frac{\gamma^{7}}{c^{2}} v a b \\
& +3 \frac{\gamma^{7}}{c^{2}} a^{3}+18 \frac{\gamma^{9}}{c^{4}} v^{2} a^{3}=0 .
\end{aligned}
$$

(4) if $\phi(\tau)=\frac{1}{c}\left(v_{0}+a_{0} \tau+\frac{1}{2} j_{0} \tau^{2}+\frac{1}{6} s_{0} \tau^{3}\right)$,

$$
\begin{aligned}
& a_{\mathrm{co}}=\gamma^{3} a=a_{0}+j_{0} \tau+\frac{1}{2} s_{0} \tau^{2}, \\
& b_{\mathrm{co}}=\gamma^{4} b+3 \frac{\gamma^{6}}{c^{2}} v a^{2}=j_{0}+s_{0} \tau,
\end{aligned}
$$

$$
\begin{aligned}
h_{\mathrm{co}}= & \gamma^{5} h+10 \frac{\gamma^{7}}{c^{2}} v a b \\
& +3 \frac{\gamma^{7}}{c^{2}} a^{3}+18 \frac{\gamma^{9}}{c^{4}} v^{2} a^{3}=s_{0},
\end{aligned}
$$

and so on.

Notice that $v=c \tanh \phi(\tau)$ cannot be explicitly integrated with respect to $t$ to obtain $x(t)$. An implicit solution is given in [5] (see also [6]) as

$$
\begin{aligned}
& x(\tau)=x_{0}+c \int_{0}^{\tau} \sinh \phi(s) \mathrm{d} s, \\
& t(\tau)=\int_{0}^{\tau} \cosh \phi(s) \mathrm{d} s .
\end{aligned}
$$

The integrals on the right-hand sides can only be computed in terms of elementary functions, and inverted, for $\phi$ up to degree 1 in $\tau$.
[1] E. L. Hill, Phys. Rev. 67, 358 (1945).

[2] W. Rindler, Phys. Rev. 119, 2082 (1960).

[3] W. G. Unruh, Phys. Rev. D 14, 870 (1976).

[4] M. Dunajski and G. Gibbons, Classical Quantum Gravity 25, 235012 (2008).

[5] J. G. Russo and P. K. Townsend, J. Phys. A 42, 445402 (2009).

[6] J. M. Pons and F. de Palol, arXiv:1811.06267.

[7] P. J. Olver, Applications of Lie Groups to Differential Equations (Springer, New York, 1993).

[8] T. M. Rocha Filho and A. Figueiredo, Comput. Phys. Commun. 182, 467 (2011).

[9] C. Duval and P. Horvathy, J. Phys. A 44, 335203 (2011).

[10] J. Gomis and K. Kamimura, Phys. Rev. D 85, 045023 (2012).

[11] M. Henkel, Phys. Rev. Lett. 78, 1940 (1997).
[12] J. Negro, M. A. del Olmo, and A. Rodríguez-Marco, J. Math. Phys. (N.Y.) 38, 3786 (1997).

[13] J. Negro, M. A. del Olmo, and A. Rodríguez-Marco, J. Math. Phys. (N.Y.) 38, 3810 (1997).

[14] E. Bergshoeff, J. Gomis, and G. Longhi, Classical Quantum Gravity 31, 205009 (2014).

[15] H. Bacry and J.-M. Lévy-Leblond, J. Math. Phys. (N.Y.) 9, 1605 (1968).

[16] J. Lukierski, P. C. Stichel, and W. J. Zakrzewski, Phys. Lett. B 650, 203 (2007).

[17] S. Fedoruk, E. Ivanov, and J. Lukierski, Phys. Rev. D 83, 085013 (2011).

[18] A. Galajinsky and I. Masterov, Nucl. Phys. B866, 212 (2013).

[19] P. D. Alvarez, J. Gomis, K. Kamimura, and M. S. Plyushchay, Ann. Phys. (Amsterdam) 322, 1556 (2007).

[20] T. Christodoulakis, N. Dimakis, and P. A. Terzis, J. Phys. A 47, 095202 (2014). 